\title{
Editorial: Nanotechnology for Precision Cancer Therapy: Advances in Gene Therapy, Immunotherapy, and 3D Bioprinting
}

\author{
Ângela Sousa $^{1 * t}$, Hernane Da Silva Barud ${ }^{2 * t}$, Zhi Ping $\mathrm{Xu}^{3 * t}$ and Christiane P. Soares ${ }^{4 * t}$ \\ ${ }^{1}$ CICS-UBI-Health Science Research Centre, University of Beira Interior, Covilhã, Portugal, ${ }^{2}$ Biopolymers and Biomaterials \\ Laboratory (BIOPOLMAT), University Center of Araraquara (UNIARA), Araraquara, Brazil, ${ }^{3}$ Australian Institute for Bioengineering \\ and Nanotechnology (AIBN), The University of Queensland, St Lucia, QLD, Australia, ${ }^{4}$ Clinical Analysis Department, School of \\ Pharmaceutical Sciences, São Paulo State University (UNESP), Araraquara, Brazil
}

Keywords: cancer theranostics, delivery systems, drugs, gene-silencing, immunotherapy, nanotechnology, phototherapy, 3D-bioprinting

\section{Editorial on the Research Topic}

\section{OPEN ACCESS}

Edited and reviewed by: Ajeet Kaushik, Florida Polytechnic University, United States

*Correspondence: Ângela Sousa angela@fcsaude.ubi.pt Hernane Da Silva Barud

hernane.barud@gmail.com Zhi Ping Xu

gordonxu@uq.edu.au

Christiane P. Soares

christiane.p.soares@unesp.br

${ }^{+}$These authors have contributed equally to this work

Specialty section:

This article was submitted to Biomedical Nanotechnology, a section of the journal

Frontiers in Nanotechnology

Received: 14 September 2021 Accepted: 29 September 2021 Published: 14 October 2021

Citation:

Sousa Â, Barud HDS, Xu ZP and Soares CP (2021) Editorial: Nanotechnology for Precision Cancer Therapy: Advances in Gene Therapy, Immunotherapy, and 3D Bioprinting.

Front. Nanotechnol. 3:776898.

doi: 10.3389/fnano.2021.776898
Nanotechnology for Precision Cancer Therapy: Advances in Gene Therapy, Immunotherapy, and 3D Bioprinting

Cancer is a significant health hazard of the 21st century, and Global Observatory of Cancer (GLOBOCAN) predicts increasing cancer incidence in the coming years. Nanotechnology has sparked a rapidly growing interest in cancer nanomedicine, promising to solve several issues associated with conventional therapeutic agents, including their poor water solubility, cargo degradation, nonspecific distribution, lack of release at the tumor site, systemic toxicity, and low therapeutic index. Thus, this Frontiers Research Topic brings relevant contributions to nanobiotechnology for cancer therapies, associating biotechnologies in genetic, epigenetic, proteomic, immunotherapy and 3D-bioprinting to current nanotechnologies tools.

Circulating tumor DNA (ctDNA) represents an emerging biomarker of liquid biopsies, contributing to the development of translational medicine for early diagnosis, therapeutic effect monitoring, and patient's prognosis with cancer (Reinert et al., 2018). However, sensitive detection of ctDNA remains a challenge, due to their short half-life and low concentrations in blood samples. Miao et al. reported a new method to address this challenge by integrating cycled enzymatic DNA amplification technique and Au nanoparticle@silicon-assisted surface-enhanced Raman scattering (SERS) technique, demonstrating the ultra-high sensitivity and specificity of the method.

RNA interference (RNAi), a post-transcriptional gene regulation mechanism, has significantly obtained high attention in cancer therapy as result of its precision to multi-targeted gene silencing. However, its direct administration has not been fruitful due to inherent lower half-lives and instability in the biological systems. Swaminathan et al. highlighted different types of nanotechnology-based delivery strategies for engineering nano-RNAi-based bio drugs, which are entering the preclinical and clinical evaluation. Promising results indicate these new combinatorial therapies can successfully and safely be used to inhibit target genes in cancer patients, shaping the future for next generation cancer therapeutics. Al-Sowayan and Al-Shareeda discussed the advantages of establishing a nanogenomics-artificial intelligence-based breast cancer diagnostic approach, compared to the gold standard radiology- or histology-based methods that are currently being adapted to artificial intelligence. Furthermore, they highlighted advantages of building the diagnostic and prognostic biomolecular profiles for breast cancers based on the exosome encapsulated content, instead of the free circulating microRNA and other biomolecules.

Brar et al. focused on the recent advancement of nanotechnology to colorectal cancer (CRC) diagnosis and treatment. The application of light-sensitive photosensitizer drugs loaded gold and 
silica nanoparticles can be successfully used for CRC diagnosis and targeted delivery of anti-cancer drugs to kill CRC cells. In addition, Perumal et al. discussed the presence of gut microbiota influences the route of biomarker detection and the route of the interaction of nanoparticle/drug complexes with target cells. Thereby, the development of nanoparticles with appropriate sizes, morphologies, chemical compositions and concentrations might overcome this fundamental barrier for the CRC diagnosis and treatment.

Nanotechnology can be a powerful approach to overcome the hydrophobicity and low aqueous solubility issues of anti-parasite/ worm drugs such as the benzimidazole (BMZ) family. Movahedi et al. developed a nanocrystal lipid-coated calcium phosphate system, which enhance BMZ solubility, specifically targeting melanoma cells, as an optimistic anti-cancer therapy. Silva et al. established a new third-generation photosensitizer, consisting in hybrid nanostructures of lipid carriers coated with porphyrin-chitosan. They demonstrated that physical and chemical properties of nanoparticles are relevant to improve the porphyrin photodynamic activity in bladder cancer cells.

Among different cancers, lung tumors has low response to therapy and new therapeutic approaches have been developed, especially against primary tumors and secondary lung metastasis (Howlader et al., 2020). Donkor and Jones explored the pulmonary route as an attractive drug delivery pathway to treat lung tumors and discuss the potential of nanotechnology to delivery cancer vaccine vectors to prevent the seeding of tumors in the lung. Ahmad et al. reviewed the interactions between an approved cancer nanomedicine with tumor microenvironment immunology and discussed the challenges that need to be addressed for the full clinical potential of ongoing cancer nanomedicines despite the encouraging preclinical data.

Recently, spheroids and organoids have been largely explored as 3D solid tumor models for recreating in vitro tumorigenesis, being considered as suitable models for drug assessment and highthroughput screening (Jensen and Teng, 2020). The main advantages of 3D bioprinting are its ability to engineer complex and controllable 3D tissue models in a higher resolution (Datta et al., 2018). Although 3D bioprinting represents a promising technology, main challenges need to be addressed to improve the results in cancer research. Kronemberger et al. explored the principal cell components and extracellular matrix composition to reconstruct the solid tumor's microenvironment, the recapitulation of in vitro tumorigenesis through

\section{REFERENCES}

Datta, P., Barui, A., Wu, Y., Ozbolat, V., Moncal, K. K., and Ozbolat, I. T. (2018). Essential Steps in Bioprinting: From Pre- to post-bioprinting. Biotechnol. Adv. 36, 1481-1504. doi:10.1016/j.biotechadv.2018.06.003

Howlader, N., Noone, A. M., Krapcho, M., Miller, D., Brest, A., and Yu, M. (2020). SEER Cancer Statistics Review, 1975-2017. Bethesda, MD: National Cancer Institute.

Jensen, C., and Teng, Y. (2020). Is it Time to Start Transitioning from 2D to 3D Cell Culture? Front. Mol. Biosci. 7, 33. doi:10.3389/ fmolb.2020.00033

Reinert, T., Henriksen, T. V., Rasmussen, M. H., Sethi, H., Sharma, S., Wu, H. T., et al. (2018). Personalized Circulating Tumor DNA Analysis to Monitor Colorectal Cancer. Cancer Res. 78, 1590. doi:10.1158/15387445 spheroids and organoids 3D models as well as the opportunities, challenges, and applications of 3D bioprinting in cancer research.

Overall, this collection of articles covers recent advances and contributes to the research topic with innovative designs and concepts in multifunctional and intelligent nanomedicine. The bionano engineered approach is not only the key strategy to overcome the intrinsic limitations of nanomaterials but also providing new aspects of the nanotechnology role in diagnostic, delivering drugs for enhancing the cancer therapy, as well as reducing drugs side effects. Therefore, nanotechnology is a promising approach to offer new opportunities of patient's treatment against cancer. The editors hope that the selected articles on this Research Topic will inspire future work to further advance to drug delivery through specific nanomaterials at the tumor sit, offering an efficient and personalized cancer therapy. In addition, nanotechnology could motivate basic and clinical research and industry support to accelerate the clinical trials implementation and approval in this field.

\section{AUTHOR CONTRIBUTIONS}

All authors listed have made a substantial, direct and intellectual contribution to the work, and approved it for publication.

\section{FUNDING}

CS was financed in part by the Coordenação de Aperfeiçoamento de Pessoal de Nível Superior-Brasil (CAPES)-Finance Code 001 and Conselho Nacional de Desenvolvimento Científico e Tecnológico (CNPq). ÂS was financed in part by the Foundation for Science and Technology (FCT), through funds from the State Budget, and by the European Regional Development Fund (ERDF), under the Portugal 2020 Program, through the Regional Operational Program of the Center (Centro 2020), through the Project with the reference UIDB/00709/2020.

\section{ACKNOWLEDGMENTS}

We thank all the authors and reviewers for their contribution to the Research Topic.

Conflict of Interest: The authors declare that the research was conducted in the absence of any commercial or financial relationships that could be construed as a potential conflict of interest.

Publisher's Note: All claims expressed in this article are solely those of the authors and do not necessarily represent those of their affiliated organizations, or those of the publisher, the editors and the reviewers. Any product that may be evaluated in this article, or claim that may be made by its manufacturer, is not guaranteed or endorsed by the publisher.

Copyright (C) 2021 Sousa, Barud, Xu and Soares. This is an open-access article distributed under the terms of the Creative Commons Attribution License (CC BY). The use, distribution or reproduction in other forums is permitted, provided the original author(s) and the copyright owner(s) are credited and that the original publication in this journal is cited, in accordance with accepted academic practice. No use, distribution or reproduction is permitted which does not comply with these terms. 